\title{
Studying Neutrino Oscillations and Searches for BSM Physics with Atmospheric Neutrinos in DUNE
}

\author{
Aaron Higuera*i \\ University of Houston \\ E-mail: ahiguera@central.uh.edu
}

The 40kt DUNE Far Detector, located at the Sanford Underground Research Facility, will offer unique capabilities to study atmospheric neutrinos. Due to the detector's excellent energy resolutions, angular resolutions, and particle ID capabilities, atmospheric neutrino analyses in DUNE can provide valuable information about 3-flavor oscillations, despite the relatively modest statistics. These data provide a complementary analysis approach to beam neutrinos, and can help in resolving ambiguities in beam-only analyses. In this paper we show the prospects on the determination of the mass hierarchy, octant of $\theta_{23}$, and measurement of $\delta_{C P}$ using atmospheric neutrinos in DUNE. Atmospheric neutrinos can also lead to a variety of beyond the standard model scenarios for neutrino transitions, propagation, and interactions. Examples that have been previously studied include CPT violation, Lorentz invariance violation, non-standard interactions, mass varying neutrinos and sterile neutrinos.

The European Physical Society Conference on High Energy Physics

5-12 July, 2017

Venice

* Speaker.

${ }^{\dagger}$ On behalf of the DUNE Collaboration. 


\section{Deep Underground Neutrino Experiment}

The Deep Underground Neutrino Experiment (DUNE) is a leading-edge neutrino experiment, DUNE will consist of two neutrino detectors that would use the world's most intense neutrino beam, the Long-Baseline Neutrino Facility (LBNF) at Fermi National Accelerator Laboratory (FNAL). The near detector will be placed near the beam's origin at FNAL and its far detector will comprise four 10kt fiducial mass liquid argon (LAr) placed deep underground (4300 mwe) at the Sanford Underground Research Laboratory in Lead, SD, 1,300 kilometers downstream of the beam's source. DUNE's far detectors will consist of several time projection chambers (TPC) that will provide excellent energy and angular resolution and great particle ID capabilities to measure the properties of neutrino interactions, in addition, with such a large detector located deep underground will also enable non-beam physics measurements, including atmospheric neutrinos, neutrinos from supernova, and searches for proton decay.

\section{Physics with Atmospheric Neutrinos in DUNE}

Atmospheric neutrinos are produced in the upper atmosphere covering a wide range of $L / E$ and with all flavors of neutrinos and antineutrinos present. Evidence of neutrino oscillation using atmospheric neutrinos was first reported in Ref. [1] and described by the deficit of muon to electron neutrinos, followed by a second measurement of a significant up-down asymmetry of high energy muon neutrino events reported in Ref. [2] and confirming the previous result later by an independent data set in Ref. [3]. Indicating that atmospheric neutrinos provide a unique tool to study neutrino oscillation complementarity to measurements performed with a neutrino beam. The DUNE far detector placed deep underground and with its large mass, is an ideal tool to perform these studies. In this paper we discuss sensitivity studies for oscillation parameters using atmospheric neutrinos in DUNE.

Fluxes of each neutrino species were computed at the far detector location, after oscillation. Interactions in the LAr medium were simulated with the GENIE event generator. We classified events as fully contained (FC) or partially contained (PC) by placing the vertex inside the detector and tracking the lepton until it reaches the edge of the detector, to take into account detector effects, detection thresholds and energy resolutions were applied to the outgoing particles, a 3\% energy resolution was assumed for FC muons and $15 \%$ for PC muons and $1 \% / \sqrt{E(\mathrm{GeV})} \oplus 1 \%$ for electrons [4]. Event rates including oscillations in $350 \mathrm{kt}$-year exposure are $\sim 14 \mathrm{k}$ FC electron-like events, $\sim 20 \mathrm{k}$ FC muon-like events and $\sim 6.8 \mathrm{k}$ PC muon-like events according with the studies presented in Ref. [5].

The oscillated flux is sensitive to matter effects when traversing through the Earth, the MSW resonance is a process which can act to modify neutrino oscillations in matter, which influences electron neutrinos in the few-GeV energy range. The resonance occurs for $v_{e}$ in the case of normal mass hierarchy ( $\mathrm{NH}, \Delta m_{32}^{2}>0$ ), and for $\bar{v}_{e}$ in the case of inverted mass hierarchy (IH, $\Delta m_{32}^{2}<$ 0). Although DUNE's far detector will not be magnetized, TPC's technology will provide 3D event imaging with excellent spatial and energy resolution offering possibilities for tagging features of events that provide statistical discrimination between neutrinos and antineutrinos. Figure 1 shows the mass hierarchy (MH) sensitivity as a function of the fiducial exposure according to 
studies performed in Ref. [5] with oscillation sensitivities calculated using a joint fit to $v_{\mu}$-like and $v_{e}$-like spectra. Unlike for beam measurements $\mathrm{MH}$ sensitivity with atmospheric neutrinos is nearly independent of the CP-violating phase. The sensitivity comes from both electron neutrino appearance as well as muon neutrino disappearance, and is strongly dependent on the true value of $\theta_{23}$.
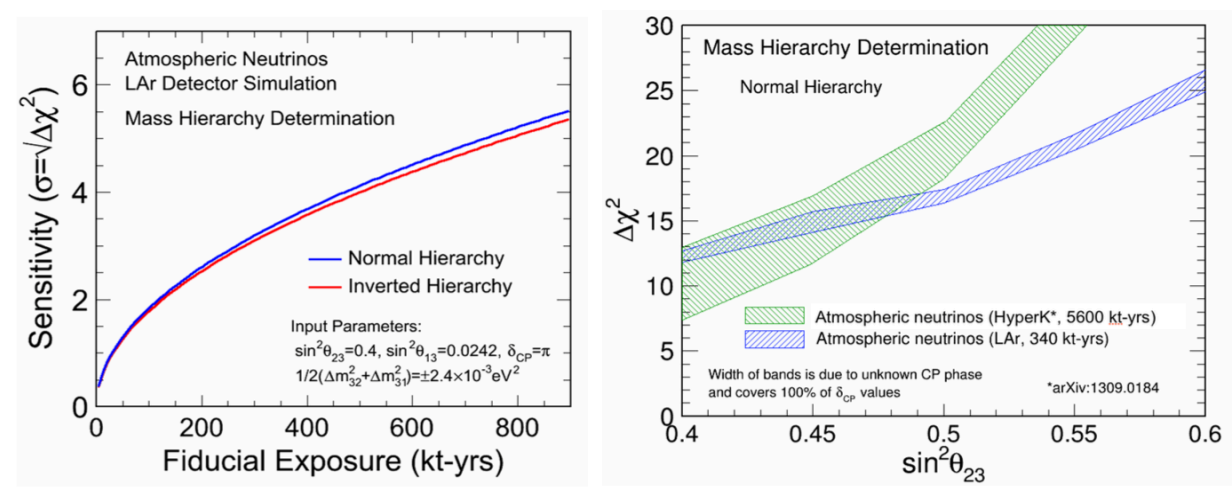

Figure 1: Sensitivity to mass hierarchy using atmospheric neutrinos as a function of fiducial exposure in a liquid argon detector (left), and as a function of the true value of $\theta_{23}$ (right). Figure taken from Ref. [5].

Figure 2 shows the octant and CPV sensitivity from a $340 \mathrm{kt} \cdot y e a r$ exposure of atmospheric neutrino data alone with early studies of a LAr detector such studies were reported in Ref. [4]. For the determination of the octant of $\theta_{23}$, the $\Delta \chi^{2}$ value is calculated between the best-fit points in the lower $\left(\theta_{23}<45^{\circ}\right)$ and higher $\left(\theta_{23}>45^{\circ}\right)$ octants, where at each, the nuisance parameters have been marginalized. The discontinuities in the slopes of the octant sensitivity plot are real features, indicating points at which the best fit moves from one hierarchy to the other. For the detection of CP violation, the $\Delta \chi^{2}$ exclusion is similarly computed for $\delta \mathrm{CP}=(0, \pi)$.
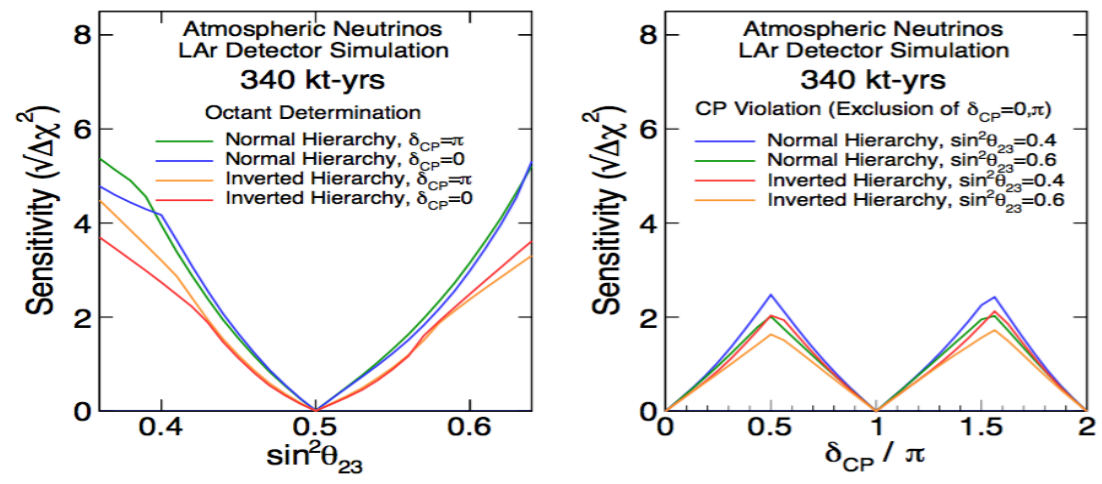

Figure 2: Sensitivity to $\theta_{23}$ octant (left) and CPV (right) using atmospheric neutrinos. Figure taken from Ref. [4].

Atmospheric neutrinos oscillated over a long distance and provide a window into a range of new physics acting as precise interferometer with high sensitivity to very small perturbations caused 
by new physics scenarios. Such potential scenarios are CPT and Lorentz invariance violation [6, 7], non-standard interactions [8], mass-varying neutrinos [9] and sterile neutrinos [10].

Furthermore atmospheric neutrino interactions in the detector represent a possible background to nucleon decay searches due to reconstruction failures, therefore studying such interactions will provide a useful sample for the development of reconstruction software and analysis. As we discussed, atmospheric neutrinos provide a complementary analysis approach to beam neutrinos, and can help resolve ambiguities in beam-only analyses enabling precision measurements of oscillation parameters.

\section{References}

[1] Y. Fukuda et al., (Super-Kamiokande Collaboration), Evidence for Oscillation of Atmospheric Neutrinos, Phys. Rev. Lett. 81, 1562 (1998).

[2] Y. Fukuda et al., (Super-Kamiokande Collaboration), Study of the atmospheric neutrino flux in the multi-GeV energy range, Phys. Lett. B436, 33 (1998).

[3] Y. Fukuda et al., (Super-Kamiokande Collaboration), Measurement of the Flux and Zenith-Angle Distribution of Upward Throughgoing Muons by Super-Kamiokande, Phys. Lett. 82, 2644 (1999).

[4] C. Adams et al., (LBNE Collaboration), The Long-Baseline Neutrino Experiment: Exploring Fundamental Symmetries of the Universe, arXiv:1307.7335 [hep-ex].

[5] R. Acciarri et al., (DUNE Collaboration), Long-Baseline Neutrino Facility (LBNF) and Deep Underground Neutrino Experiment (DUNE) Conceptual Design Report Volume 2: The Physics Program for DUNE at LBNF, arXiv:1512.06148 [physics.ins-det].

[6] V. A. Kostelecky and M. Mewes, Lorentz and CPT violation in neutrinos, Phys. Rev. D69, 016005, (2004).

[7] A. Kostelecky and M. Mewes, Neutrinos with Lorentz-violating operators of arbitrary dimension, Phys. Rev. D85, 096005, (2012).

[8] A. Chatterjee, P. Mehta, D. Choudhury, and R. Gandhi, Testing nonstandard neutrino matter interactions in atmospheric neutrino propagation, Phys. Rev. D 93, 093017 (2016).

[9] K. Abe et al., (Super-Kamiokande Collaboration), Search for Matter-Dependent Atmospheric Neutrino Oscillations in Super-Kamiokande, Phys. Rev. D77, 052001, (2008).

[10] K. Abe et al., (Super-Kamiokande Collaboration), Limits on sterile neutrino mixing using atmospheric neutrinos in Super-Kamiokande, Phys. Rev. D91, 052019, (2015). 